\title{
Obliquely propagating large amplitude solitary waves in charge neutral plasmas
}

\author{
F. Verheest ${ }^{1,2}$ \\ ${ }^{1}$ Sterrenkundig Observatorium, Universiteit Gent, Krijgslaan 281, 9000 Gent, Belgium \\ ${ }^{2}$ School of Physics, University of KwaZulu-Natal, Private Bag X54001, Durban 4000, South Africa
}

Received: 27 November 2006 - Revised: 24 January 2007 - Accepted: 24 January 2007 - Published: 30 January 2007

\begin{abstract}
This paper deals in a consistent way with the implications, for the existence of large amplitude stationary structures in general plasmas, of assuming strict charge neutrality between electrons and ions. With the limit of pair plasmas in mind, electron inertia is retained. Combining in a fluid dynamic treatment the conservation of mass, momentum and energy with strict charge neutrality has indicated that nonlinear solitary waves (as e.g. oscillitons) cannot exist in electron-ion plasmas, at no angle of propagation with respect to the static magnetic field. Specifically for oblique propagation, the proof has turned out to be more involved than for parallel or perpendicular modes. The only exception is pair plasmas that are able to support large charge neutral solitons, owing to the high degree of symmetry naturally inherent in such plasmas. The nonexistence, in particular, of oscillitons is attributed to the breakdown of the plasma approximation in dealing with Poisson's law, rather than to relativistic effects. It is hoped that future space observations will allow to discriminate between oscillitons and large wave packets, by focusing on the time variability (or not) of the phase, since the amplitude or envelope graphs look very similar.
\end{abstract}

\section{Introduction}

Plasmas are an intrinsically nonlinear medium that can support a great variety of diverse waves, far more than can be cited here, and discussed in many a classic textbook. During the last decade or so there have been many space observations where large amplitude spiky or wave packet-like structures have been observed, both electrostatic (Matsumoto et al., 1994; Frantz et al., 1998; Ergun et al., 1998; Cattell et al., 1999, 2003; Pottelette et al., 1999; Pickett et al., 2003, 2004) as well as electromagnetic (Paschmann et al., 1988;

Correspondence to: F. Verheest

(frank.verheest@UGent.be)
Schwartz et al., 1988; Thomsen et al., 1988; Kivelson et al., 1996; Zhang et al., 1998; Baumjohann et al., 1999; Petkaki and Dougherty, 2001; Stasiewicz et al., 2003a,b) in character. The references given here are not at all exhaustive, but represent a typical selection.

A proper theoretical description of these observations can only be fully nonlinear, given their large amplitudes. Treatments based on singular perturbation theory, leading to well known paradigms like the Korteweg-de Vries or (derivative) nonlinear Schrödinger equations, imply an iterative scheme (Ablowitz and Clarkson, 1991) that can only be trusted for nonlinearities that are not too strong. Otherwise, terms of higher order in the expansion are not small enough to be safely neglected, compared to the ones retained (Hellberg et al., 1992).

The preferred analysis of such large amplitude structures has been in terms of solitary waves which ideally propagate with an unchanging shape in dissipationless plasmas, typically as a localized hump or dip profile, known since 1834 from shallow water wave observations. Early plasma physics treatments have covered both electromagnetic (Adlam and Allen, 1958) and electrostatic (Sagdeev, 1966) structures. The Sagdeev pseudopotential analysis has been highly successful in explaining the existence regimes of electrostatic solitons and double layers in plasmas with different ion and electron compositions, mostly in terms of the classical mechanics analogy with the motion of particle in a onedimensional potential.

The electromagnetic counterparts have received less attention, presumably because the description involves more degrees of freedom, without a concomitant increase in obvious invariants like the conservation of mass, global momentum and energy. Thus there was no simple reduction to one single differential equation for one of the field variables, except in rather special cases, as reviewed by Decoster (1978) with particular emphasis on parallel and perpendicular propagation with respect to the ambient magnetic field.

Published by Copernicus GmbH on behalf of the European Geosciences Union and the American Geophysical Union. 
Recently, McKenzie and coworkers (McKenzie and Doyle, 2001; McKenzie et al., 2001; Sauer et al., 2001; Dubinin et al., 2002; McKenzie et al., 2004) have revived interest in these approaches, by focussing on the fluid dynamic interpretation of how various first integrals limit the available parameter space, rather than relying on numerical work to find these limitations, as had mostly been the case before. Especially for the full description of electromagnetic modes the obtention of several of the invariants has not always been transparent. Adopting the concept of a generalized vorticity has now allowed a much more evident derivation of these first integrals and given a physical interpretation where that was previously lacking (Mace et al., 2007).

One of the more intriguing concepts to emerge from the fluid dynamic emphasis on the description of large amplitude electromagnetic structures is that of an oscilliton, exhibiting spatial oscillations superimposed on the spatial growth and decay which is characteristic of the more usual solitons (Sauer et al., 2001, 2002; Dubinin et al., 2003; Verheest et al., 2004; Cattaert and Verheest, 2005). Although the graph looks very much like that of an envelope soliton, typical for the solutions of the (derivative) nonlinear Schrödinger equation (Hada et al., 1989), the latter only have a stationary envelope but a phase varying slowly in time, in the frame comoving with the structure.

Because of the intricacies encountered in the mathematical treatment of larger amplitude nonlinear modes, several simplifying assumptions have been made when dealing with ordinary ion-electron or bi-ion plasmas. The most common is that of charge neutrality (Adlam and Allen, 1958; Hackenberg et al., 1998; McKenzie and Doyle, 2001; Sauer et al., 2001; Dubinin et al., 2002, 2003), with the argument that one is looking at slow modes or low-frequency phenomena. However, although the concept of frequency might be borrowed from the linear counterparts of the nonlinear waves studied, it is not a well defined property of stationary modes, where the (phase) velocity is the meaningful quantity to deal with.

Consequently, attempts have been made to see how crucial the introduction of charge neutrality is for the real possibility of encountering oscillitons. It was found that one had to deal in a subtle way with Poisson's equation, by retaining the parallel wave electric field (in the mathematical limit $\varepsilon_{0} \rightarrow 0$ ), while admitting that the ion and electron densities were equal (Verheest et al., 2004), in what is known in the literature as the plasma approximation (Chen, 1974). It should nevertheless be kept in mind that this is true only for low-frequency motions where the electron inertia is not a factor (Chen, 1974). It might be questionable how far the plasma approximation can be used in the study of large stationary structures, where momentum conservation needs the contributions of both species.

Further investigations into the oscilliton concept, dealing in a more correct way with Poisson's equation, have included a fully relativistic description, leading to the conclusion that this seemed to prevent the formation of oscillitons (McKenzie et al., 2005). This was based on the numerical integration of a more complicated set of equations (owing to the absence of charge neutrality) and attributed to the fact that dispersion cannot prevent nonlinear steepening, the electron velocities growing to the speed of light.

The concept of an oscilliton has thus not found a wide acceptance in the community, also because space observations cannot (yet) discriminate between true oscillitons and envelope solitons or large amplitude wave packets. It is hence necessary to revisit the consequences of the imposition of strict charge neutrality on large amplitude stationary structures in general. By strict we will mean that Poisson's law needs to be obeyed and the parallel electric field vanishes, unlike in the plasma approximation.

Several other arguments have come together in dealing with this question. One is that if one goes to pure pair plasmas, where the charges (in absolute value) and the masses are equal, there are no oscillitons but one obtains a traditional looking soliton, without varying phase (Verheest and Cattaert, 2004, 2005).

Pair plasmas have been studied with specific astrophysical applications in mind, like pulsar magnetospheres, where the intense energies give rise to electron-positron pair creation and annihilation (Pacini, 1968; Goldreich and Julian, 1969; Ostriker and Gunn, 1969; Rees, 1971; Sturrock, 1971). On the other hand, experiments on electron-positron plasmas have been performed in rather demanding circumstances, given the ready annihilation of the electrons and the positrons (Surko et al., 1989; Boehmer et al., 1995). The study of wave modes in pair plasmas has recently been rekindled by experiments in fullerene pair plasmas, where $\mathrm{C}_{60}^{ \pm}$were produced in equal numbers, and a fullerene pair plasma is a way of mimicking electron-positron plasma behaviour, without having to worry about fast annihilation (Oohara and Hatakeyama, 2003).

It is worth recalling that for the usual plasma compositions, the great disparities in mass between the negative and positive charge carriers induce quite different time and length scales that can be advantageously exploited to disentangle some of the wave characteristics, specially for linear modes. In this respect pair plasmas are radically different, and this is already evident at the linear level where e.g. at propagation parallel to a static magnetic field the transverse modes are no longer circularly polarized as in electron-ion plasmas (Stix, 1992) but degenerate into modes that are linearly polarized in any direction perpendicular to the static field (Iwamoto, 1993; Zank and Greaves, 1995).

Another argument can be found in very recent work where the use of generalized Beltrami fields and demanding that the fluid velocities are parallel to their generalized vorticities allows one to decompose the nonlinear description into a set of coupled linear vector equations. Because of their linear character, the latter admit harmonic waves that are circularly polarized in ordinary plasmas, but reduce to linearly polarized 
structures in pure pair plasmas (Z. Yoshida, personal communication, 2006; S. Mahajan, personal communication, 2006), and this without any linearization of the basic equations in the traditional sense of a small amplitude treatment.

Hence, we will investigate what consequences charge neutrality really has, when taken at strict face value, and this in the model of an electron-ion plasma, where the charge carriers have elementary charges of opposite sign and masses which are different, in principle, but can include the pair plasma limit of equal masses. With the apparent dichotomy between ordinary and pair plasmas in mind, we cannot neglect electron inertia, in particular, as has been done in some of the earlier papers (McKenzie and Doyle, 2001). We are allowed to consider for simplicity a cold plasma model, because several of the interesting electromagnetic nonlinear structures do not specifically rely on thermal pressure effects, which is in particular the case for the oscilliton concept.

The paper is structured as follows. After this introduction, Sect. 2 deals with the formalism and invariants used, and gives already a preliminary discussion of the consequences of charge neutrality for the existence conditions of large amplitude modes, at parallel and perpendicular propagation. Section 3 addresses the nonexistence of solitons or oscillitons in the usual electron-ion plasmas, specifically for oblique propagation, as the proof is rather harder than for purely parallel or perpendicular modes. Section 4 sums up the conclusions, while Appendix A discusses the possibility of having linear evanescent waves, as a prelude to the construction of fully nonlinear solitary waves. Finally, Appendix B recalls some elements of the large amplitude treatment for pair plasmas.

\section{Formalism and invariants}

\subsection{Model}

For waves propagating along the $\mathrm{x}$-axis of a reference frame, we can, without loss of generality, orient the $x, z$ plane so that it contains the static magnetic field $\boldsymbol{B}_{0}=B_{x 0} \boldsymbol{e}_{x}+B_{z 0} \boldsymbol{e}_{z}$. The cold plasma equations include the continuity and momentum equations,

$$
\begin{aligned}
& \frac{\partial n_{j}}{\partial t}+\frac{\partial}{\partial x}\left(n_{j} v_{j x}\right)=0, \\
& \frac{\partial \boldsymbol{v}_{j}}{\partial t}+v_{j x} \frac{\partial \boldsymbol{v}_{j}}{\partial x}=\frac{q_{j}}{m_{j}}\left(\boldsymbol{E}+\boldsymbol{v}_{j} \times \boldsymbol{B}\right),
\end{aligned}
$$

where $n_{j}$ refers to the number densities of the positive ions or positrons $(j=i)$ and of the negative ions or electrons $(j=e)$, having charges $q_{j}$ and masses $m_{j}$. For simplicity, we assume that both species have unit charge $e$, so that $q_{i}=+e$ and $q_{e}=-e$. This can always be achieved by a suitable redefinition of the masses and number densities. Furthermore, $\boldsymbol{v}_{j}$ refers to the respective fluid velocities, while $\boldsymbol{E}$ and $\boldsymbol{B}$ are the (total) electric and magnetic fields, respectively. The system is closed by Maxwell's equations,

$$
\begin{aligned}
& \boldsymbol{e}_{x} \times \frac{\partial \boldsymbol{E}}{\partial x}+\frac{\partial \boldsymbol{B}}{\partial t}=\mathbf{0}, \\
& \boldsymbol{e}_{x} \times \frac{\partial \boldsymbol{B}}{\partial x}=\frac{1}{c^{2}} \frac{\partial \boldsymbol{E}}{\partial t}+\mu_{0} e\left(n_{i} \boldsymbol{v}_{i}-n_{e} \boldsymbol{v}_{e}\right), \\
& \varepsilon_{0} \frac{\partial E_{x}}{\partial x}=e\left(n_{i}-n_{e}\right) .
\end{aligned}
$$

The parallel magnetic field $B_{x}=B_{x 0}$ is constant.

\subsection{Nonlinear modes and first integrals}

The linear modes in such plasmas have been extensively studied and discussions can be found in standard textbooks. Here we address the role of charge neutrality for nonlinear modes that propagate in an oblique direction with respect to the static magnetic induction. The angle between the two directions is denoted by $\vartheta$.

Charge neutrality means that we impose from the outset that $n_{i}=n_{e}=n$, but $n$ might differ from its equilibrium value $n_{0}$, the modes not being assumed incompressible.

In view of the nonlinear structures to be studied we use a frame in which they appear stationary, so that all time derivatives vanish and the plasma species have an undisturbed reference speed $V$ along the $\mathrm{x}$-axis at $x=-\infty$, where also $\boldsymbol{B} \rightarrow \boldsymbol{B}_{0}$ and $\boldsymbol{E} \rightarrow \boldsymbol{E}_{0}$. Such a solitary wave Ansatz excludes the consideration of periodic nonlinear waves with constant amplitudes, which do not vanish for the undisturbed conditions at $x=-\infty$. This applies in particular to the large amplitude circularly polarized transverse modes at parallel propagation, discussed by many authors and reviewed by Decoster (1978).

All derivatives now being with respect to $x$, the continuity equations (1) express conservation of parallel (mass) flux,

$n v_{i x}=n v_{e x}=n_{0} V$,

and consequently $v_{i x}=v_{e x}=v_{x}$, annulling the parallel current in Ampère's law (4). From the stationary form of Faraday's law (3) there follows that $\boldsymbol{E}_{\perp}$ is constant, and hence, given the conditions at infinity,

$\boldsymbol{E}_{\perp}=V B_{z 0} \boldsymbol{e}_{y}$.

The subscript $\perp$ will be used for projections perpendicular to the x-axis. Poisson's equation (5) then tells us that $E_{x}=0$.

We now multiply the equations of motion (2) by $n m_{j}$ for positive and negative species, sum the two, use Ampère's law (4) and flux conservation (6), in order to obtain expressions which can be integrated with respect to $x$. This yields the parallel and perpendicular momentum invariants, respectively,

$$
\begin{aligned}
& v_{x}=V-\frac{1}{2 \mu_{0} n_{0} V\left(m_{i}+m_{e}\right)}\left(B_{\perp}^{2}-B_{z 0}^{2}\right), \\
& m_{i} \boldsymbol{v}_{i \perp}+m_{e} \boldsymbol{v}_{e \perp}=\frac{B_{x 0}}{\mu_{0} n_{0} V}\left(\boldsymbol{B}_{\perp}-B_{z 0} \boldsymbol{e}_{z}\right) .
\end{aligned}
$$


The sum of the projection of the equations of motion (2) on $n m_{j} \boldsymbol{v}_{j}$ can be integrated to give the energy integral,

$$
\begin{aligned}
& \left(m_{i}+m_{e}\right)\left(v_{x}^{2}-V^{2}\right)+m_{i} v_{i \perp}^{2}+m_{e} v_{e \perp}^{2} \\
& +\frac{2 B_{z 0}}{\mu_{0} n_{0}}\left(B_{z}-B_{z 0}\right)=0 .
\end{aligned}
$$

These first integrals have been obtained before in various guises (Decoster, 1978; McKenzie et al., 2001; Dubinin et al., 2003; Verheest et al., 2004; Mace et al., 2007).

\subsection{First consequences of strict charge neutrality}

Because of charge neutrality and its implications for the parallel velocities, the two parallel equations of motion should be compatible, in the sense that

$$
\begin{aligned}
v_{x} \frac{d v_{x}}{d x} & =\frac{e}{m_{i}}\left(\boldsymbol{v}_{i \perp} \times \boldsymbol{B}_{\perp}\right) \cdot \boldsymbol{e}_{x} \\
& =-\frac{e}{m_{e}}\left(\boldsymbol{v}_{e \perp} \times \boldsymbol{B}_{\perp}\right) \cdot \boldsymbol{e}_{x},
\end{aligned}
$$

from which it follows that

$$
\left(m_{e} \boldsymbol{v}_{i \perp}+m_{i} \boldsymbol{v}_{e \perp}\right) \times \boldsymbol{B}_{\perp}=\mathbf{0} .
$$

Note that this is different from Eq. (9) which implies that

$$
\left(m_{i} \boldsymbol{v}_{i \perp}+m_{e} \boldsymbol{v}_{e \perp}\right) \times \boldsymbol{B}_{\perp}=\frac{B_{x 0} B_{z 0}}{\mu_{0} n_{0} V} B_{y} \boldsymbol{e}_{x} .
$$

Should we assume that $m_{i}=m_{e}$, as in pure pair plasmas, then Eqs. (12) and (13) can only be compatible provided $B_{x 0} B_{z 0} B_{y}=0$. For strictly oblique propagation $(\vartheta \neq 0$ and $\vartheta \neq 90^{\circ}$ or $B_{x 0} \neq 0$ and $\left.B_{z 0} \neq 0\right)$ this requirement gives $B_{y}=0$. In the limit of parallel propagation $\left(\vartheta=0\right.$ and $B_{z 0}=0$ ) of electromagnetic modes in pair plasmas, we know that the wave magnetic field is transverse and linearly polarized (Verheest and Cattaert, 2004), and there is no harm to take it along the $\mathrm{z}$-axis, so that $B_{y}=0$ implies no loss of generality. Continuing briefly with pair plasmas, we find for all angles $\vartheta \neq 90^{\circ}$ that the magnetic field has no component outside the plane spanned by the directions of wave propagation and of the external magnetic field, and by continuity this is also expected to be the case when $\vartheta \rightarrow 90^{\circ}$. Further details of the derivation of a pseudopotential integral and the discussion of the existence regimes for obliquely propagating solitary waves in pair plasmas can be found elsewhere (Verheest and Cattaert, 2005) and have been briefly recalled in Appendix B, for the sake of readability and completeness.

Hence the remaining question to be answered here is whether $m_{i} \neq m_{e}$ can be chosen without leading to contradictions, in other words, whether assuming charge neutrality for large amplitude stationary solitary structures only works for pair plasmas or not.

Adding Eqs. (12) and (13) gives

$\left(\boldsymbol{v}_{i \perp}+\boldsymbol{v}_{e \perp}\right) \times \boldsymbol{B}_{\perp}=\frac{B_{x 0} B_{z 0}}{\mu_{0} n_{0} V\left(m_{i}+m_{e}\right)} B_{y} \boldsymbol{e}_{x}$, so that Eq. (11) then yields

$\left(m_{i}-m_{e}\right) v_{x} \frac{d v_{x}}{d x}=\frac{e B_{x 0} B_{z 0}}{\mu_{0} n_{0} V\left(m_{i}+m_{e}\right)} B_{y}$.

Taking for a moment $B_{x 0} B_{z 0} B_{y}=0$ and $m_{i} \neq m_{e}$ immediately leads to $v_{x}$ being constant $\left(v_{x}=V\right)$, and consequently from Eq. (8) also that $B_{\perp}^{2}=B_{z 0}^{2}$. Thus the hodograph of $\boldsymbol{B}_{\perp}$ is a circle in the $y, z$ plane with radius $B_{z 0}$, indicating in principle periodic solutions at constant amplitude,

$B_{y}=B_{z 0} \sin \alpha$,

$B_{z}=B_{z 0} \cos \alpha$.

Hence $\boldsymbol{B}_{\perp}$ can only obey the undisturbed conditions if $\alpha \equiv 0$, or also $B_{y} \equiv 0$ and $B_{z} \equiv B_{z 0}$. Then Eq. (10) shows that also $v_{j \perp}^{2}=0$ : all physical variables are constant and equal to their undisturbed values. Hence no solitary structures can exist for strictly parallel or perpendicular propagation or when $\boldsymbol{B}_{\perp}$ is restricted to the $x, z$ plane, which is what $B_{x 0} B_{z 0} B_{y}=0$ implies, at least for plasmas in which $m_{i} \neq m_{e}$.

\section{Nonexistence of solitary modes when $m_{i} \neq m_{e}$}

We continue now with the general case that $B_{x 0} B_{z 0} B_{y} \neq 0$ and $m_{i} \neq m_{e}$. Eliminating $\boldsymbol{v}_{e \perp}$ between Eqs. (12) and (13) yields

$\boldsymbol{v}_{i \perp} \times \boldsymbol{B}_{\perp}=\frac{m_{i} B_{x 0} B_{z 0}}{\mu_{0} n_{0} V\left(m_{i}^{2}-m_{e}^{2}\right)} B_{y} \boldsymbol{e}_{x}$,

with an analogous result for $\boldsymbol{v}_{e \perp}$. We can thus express e.g. $v_{i z}$ in terms of $v_{i y}, B_{y}$ and $B_{z}$, which we will do via an auxiliary variable $u$ that is a function of $x$, as are the other variables to be determined. This gives in vector notation that

$\boldsymbol{v}_{i \perp}=u \boldsymbol{B}_{\perp}-\frac{m_{i} B_{x 0} B_{z 0}}{\mu_{0} n_{0} V\left(m_{i}^{2}-m_{e}^{2}\right)} \boldsymbol{e}_{z}$.

This provisional way of writing the ion velocities is for mathematical convenience only and we will show that $u$ depends in an intricate way on $x$ through $B_{y}$ and $B_{z}$.

We can use Eq. (9) to express $\boldsymbol{v}_{e \perp}$ as

$$
\begin{aligned}
\boldsymbol{v}_{e \perp}= & -\frac{m_{i}}{m_{e}} u \boldsymbol{B}_{\perp}+\frac{B_{x 0}}{\mu_{0} n_{0} m_{e} V} \boldsymbol{B}_{\perp} \\
& +\frac{m_{e} B_{x 0} B_{z 0}}{\mu_{0} n_{0} V\left(m_{i}^{2}-m_{e}^{2}\right)} \boldsymbol{e}_{z} .
\end{aligned}
$$

Now substitute Eqs. (18) and (19), together with $v_{x}$ from Eq. (8), in the energy integral (10), which becomes a quadratic equation in $u$ that can be written as

$$
B_{\perp}^{2}\left(\mu_{0} n_{0} V u\right)^{2}-A_{1}\left(\mu_{0} n_{0} V u\right)+C_{1}=0 .
$$

The coefficients are given as

$$
A_{1}=2 B_{x 0}\left[\frac{B_{\perp}^{2}}{m_{i}+m_{e}}+\frac{m_{e} B_{z 0} B_{z}}{m_{i}^{2}-m_{e}^{2}}\right],
$$




$$
\begin{aligned}
C_{1}= & \frac{B_{x 0}^{2}}{m_{i}\left(m_{i}+m_{e}\right)}\left[B_{\perp}^{2}+\frac{2 m_{e}^{2} B_{z 0} B_{z}}{m_{i}^{2}-m_{e}^{2}}\right. \\
& \left.+\frac{m_{e}\left(m_{i}^{3}+m_{e}^{3}\right) B_{z 0}^{2}}{\left(m_{i}^{2}-m_{e}^{2}\right)^{2}}\right]+\frac{m_{e}\left(B_{\perp}^{2}-B_{z 0}^{2}\right)^{2}}{4 m_{i}\left(m_{i}+m_{e}\right)^{2}} \\
& -\mu_{0} n_{0} V^{2} \frac{m_{e}\left(B_{\perp}^{2}-2 B_{z 0} B_{z}+B_{z 0}^{2}\right)}{m_{i}\left(m_{i}+m_{e}\right)}
\end{aligned}
$$

and depend only on $B_{\perp}$ and $B_{z}$, or equivalently, on $B_{y}$ and $B_{z}$ in a polynomial way. Solving Eq. (20) formally for $u$ in terms of the magnetic field components $B_{y}$ and $B_{z}$ yields unwieldy formulae that bring no immediate physical insight.

Since the velocities have thus been determined in terms of $B_{y}$ and $B_{z}$, at least in principle, one might think that the two components of the perpendicular Ampère's law (4) then give the evolution of the magnetic field and the problem is done.

However, we still have to check that the velocities thus obtained satisfy also the perpendicular equations of motion (2), which we write, e.g., for the ions in a rather hybrid notation as

$$
\begin{aligned}
v_{x} \boldsymbol{B}_{\perp} \frac{d u}{d x}= & \frac{e}{m_{i}}\left(V B_{z 0} \boldsymbol{e}_{y}+v_{x} \boldsymbol{e}_{x} \times \boldsymbol{B}_{\perp}+B_{x 0} \boldsymbol{v}_{i \perp} \times \boldsymbol{e}_{x}\right) \\
& -e \mu_{0} n_{0} V u\left(\boldsymbol{v}_{i \perp}-\boldsymbol{v}_{e \perp}\right) \times \boldsymbol{e}_{x} .
\end{aligned}
$$

On the r.h.s. we have used Ampère's law (4) to write $v_{x} d \boldsymbol{B}_{\perp} / d x$ in terms of the species' velocities, the latter involving $u$ in a linear fashion. It is now easy to eliminate the as yet unknown expression $d u / d x$ by cross multiplying Eq. (22) by $\boldsymbol{B}_{\perp}$, which yields another quadratic equation for $u$, namely

$B_{\perp}^{2}\left(\mu_{0} n_{0} V u\right)^{2}-A_{2}\left(\mu_{0} n_{0} V u\right)+C_{2}=0$,

with coefficients given by

$$
\begin{aligned}
A_{2}= & B_{x 0}\left[\frac{B_{\perp}^{2}}{m_{i}}+\frac{m_{e} B_{z 0} B_{z}}{m_{i}^{2}-m_{e}^{2}}\right], \\
C_{2}= & \mu_{0} n_{0} V^{2} \frac{m_{e}\left(B_{\perp}^{2}-B_{z 0} B_{z}\right)}{m_{i}\left(m_{i}+m_{e}\right)}-\frac{m_{e} B_{\perp}^{2}\left(B_{\perp}^{2}-B_{z 0}^{2}\right)}{2 m_{i}\left(m_{i}+m_{e}\right)^{2}} \\
& +\frac{m_{e} B_{x 0}^{2} B_{z 0} B_{z}}{\left(m_{i}+m_{e}\right)^{2}\left(m_{i}-m_{e}\right)} .
\end{aligned}
$$

Both Eqs. (20) and (23) have to give the same, acceptable expression for $u$. To have an idea of where this is leading to, we first take the undisturbed limits for $B_{y} \rightarrow 0$ and $B_{z} \rightarrow B_{z 0}$, which gives from Eqs. (20) and (23) that

$$
\begin{aligned}
& \left(\mu_{0} n_{0} V u_{\infty}-\frac{m_{i} B_{x 0}}{m_{i}^{2}-m_{e}^{2}}\right)^{2}=0, \\
& \left(\mu_{0} n_{0} V u_{\infty}-\frac{m_{i} B_{x 0}}{m_{i}^{2}-m_{e}^{2}}\right) \times \\
& \times\left(\mu_{0} n_{0} V u_{\infty}-\frac{m_{e} B_{x 0}}{m_{i}\left(m_{i}+m_{e}\right)}\right)=0 .
\end{aligned}
$$

The new notation $u_{\infty}$ stands for the limiting value of $u$ and can be obtained from Eq. (18) as

$u_{\infty}=\frac{m_{i} B_{x 0}}{\mu_{0} n_{0} V\left(m_{i}^{2}-m_{e}^{2}\right)}$.

As Eq. (25) clearly shows, the two quadratic equations are such that neither of the two is redundant, and both have to be considered. As only one of the two roots of Eq. (23) has the right limit, we have to express the fact that that root is also a solution of Eq. (20). The condition for that to happen is that the resultant of the two (quadratic) equations vanishes (Rektorys, 1969),

$$
\left|\begin{array}{cccc}
B_{\perp}^{2} & -A_{1} & C_{1} & 0 \\
0 & B_{\perp}^{2} & -A_{1} & C_{1} \\
B_{\perp}^{2} & -A_{2} & C_{2} & 0 \\
0 & B_{\perp}^{2} & -A_{2} & C_{2}
\end{array}\right|=0
$$

This involves only $B_{y}$ and $B_{z}$, besides various parameters, so that the resultant is an algebraic, even polynomial relation, leading, in principle, to $B_{y}=f\left(B_{z}\right)$, or something equivalent, however complicated the actual expression might be. In all these steps the $x$ dependence is through $B_{y}$ and $B_{z}$. This means that the acceptable, common root $u$ of Eqs. (20) and (23) is also expressible, again in principle, as a function of $B_{z}$, with analogous consequences for all the perpendicular velocities.

Combining now the projections of the perpendicular Ampère's law and eliminating $d B_{z} / d x$ gives

$$
\begin{aligned}
& B_{z}\left[\mu_{0} n_{0} V u\left(B_{z}\right)-\frac{B_{x 0}}{m_{i}+m_{e}}\right]\left[1+f\left(B_{z}\right) \frac{d f}{d B_{z}}\left(B_{z}\right)\right] \\
& =\frac{m_{e} B_{x 0} B_{z 0}}{m_{i}^{2}-m_{e}^{2}} .
\end{aligned}
$$

Abbreviating the 1.h.s. by $F\left(B_{z}\right)$, Eq. (28) shows that $F\left(B_{z}\right)$ is constant, where we expected a relation that should give $B_{z}(x)$. Using the chain rule for the derivative of Eq. (28) gives

$\frac{d F}{d B_{z}} \frac{d B_{z}}{d x}=0$,

so that either $F\left(B_{z}\right)$ cannot contain $B_{z}$, which patently is not the case, or $B_{z}(x)$ is constant in $x$ and given in terms of (constant) parameters. Note that we still obtain from Eq. (28) the correct $u_{\infty}$ in the limit $B_{z} \rightarrow B_{z 0}$ and $B_{y}=f\left(B_{z}\right) \rightarrow 0$, and the latter limit is the only possible solution left.

Because we have supposed for this subsection that $B_{x 0} B_{z 0} B_{y} \neq 0$ and that $m_{i} \neq m_{e}$, we have clearly arrived at a dead end: no solitary waves are possible in strictly charge neutral plasmas with $m_{i} \neq m_{e}$. 


\section{Conclusions}

Because of the difficulties cropping up in the mathematical treatment of larger amplitude nonlinear modes, several simplifying assumptions are encountered in the literature, when dealing with ordinary ion-electron or bi-ion plasmas. One of these is charge neutrality, with the argument that one is looking at slow modes or low-frequency phenomena. Attempts to see how crucial this hypothesis is have centered on the plasma approximation in dealing with Poisson's equation, specifically when studying oscillitons (Verheest et al., 2004). Moreover, a fully relativistic description prevents the formation of oscillitons, and this is attributed to the fact that dispersion cannot prevent nonlinear steepening, the electron velocities quickly increasing to the speed of light (McKenzie et al., 2005).

We have revisited in the present paper, in a consequent way, what strict charge neutrality implies for the existence of large amplitude stationary structures in general, and with the dichotomy between ordinary and pair plasmas in mind, we have kept full electron inertia. Combining the existing mass, momentum and energy invariants with strict charge neutrality has indicated that solitons and oscillitons cannot exist in electron-ion plasmas, at no angle of propagation with respect to the static magnetic field. Specifically for oblique propagation, the proof has turned out to be rather more convoluted than for purely parallel or perpendicular modes. The only exception is pair plasmas, which are able, in a natural way, to support charge neutral solitons, in view of the high degree of symmetry inherent in such plasmas (Verheest and Cattaert, 2005).

It would thus seem that the disappearance of oscillitons in a relativistic electron-ion plasma (McKenzie et al., 2005) is a consequence of the breakdown of the plasma approximation, rather than of the relativistic description per se. Only when the plasma approximation is reasonably obeyed can the concept of oscillitons be salvaged, and this probably not for too large structures (Cattaert and Verheest, 2005). It is thus hoped that more detailed future observations of large scale nonlinear phenomena in space will allow us to discriminate between oscillitons and large wave packets, by focusing on the time variability (or not) of the phase, since the amplitude or envelope graphs look very similar. Present day measurements are not able to do so.

\section{Appendix A}

\section{Evanescent linear modes}

When looking for regions in parameter space where nonlinear solitons and/or oscillitons might be found, it is interesting to understand what a linearized description tells us. This can be seen in the present context as follows. Linearizing the basic equations (1)-(5) around the undisturbed values, putting $\partial \cdot / \partial t=0$ and supposing that all perturbations vary as $f=\delta f \exp (\kappa x)$, gives a series of algebraic equations that can easily be solved. The condition to find nontrivial solutions is that the linear dispersion law vanishes, and this might occur for $\kappa$ real (evanescent solutions), imaginary (periodic harmonic solutions) or complex (growing or decaying solutions accompanied by superimposed oscillations).

The waves of the evanescent type are growing or decaying at the end of the structure and this could give rise to solitons if the nonlinearity can be balanced by dispersive effects. For that, of course, the full nonlinear equations have to be considered. Similarly, complex $\kappa$ values could point to the existence of oscillitons under the proper conditions. It is thus seen that solitons and oscillitons could occur in regions of parameter space where linear harmonic waves cannot propagate (Hackenberg et al., 1998; McKenzie et al., 2001; Dubinin et al., 2002).

Hence, imposing charge neutrality in the linearized versions of Eq. (5) and using Eq. (3) in the same small amplitude limit indicates that $\delta \boldsymbol{E}=\mathbf{0}$ and of course we also have that $\delta B_{x}=0$.

The linearized equations of motions (2) give that

$$
\begin{aligned}
\delta v_{j x} & =\frac{\Omega_{j} \sin \vartheta}{\kappa V} \delta v_{j y}, \\
\delta v_{j y} & =\frac{\Omega_{j} \cos \vartheta}{\kappa V} \delta v_{j z}-\frac{\Omega_{j} \sin \vartheta}{\kappa V} \delta v_{j x}-\frac{\Omega_{j}}{\kappa B_{0}} \delta B_{z}, \\
\delta v_{j z} & =\frac{\Omega_{j}}{\kappa B_{0}} \delta B_{y}-\frac{\Omega_{j} \cos \vartheta}{\kappa V} \delta v_{j y},
\end{aligned}
$$

where we have introduced the algebraic gyrofrequency $\Omega_{j}=q_{j} B_{0} / m_{j}$ per species, including the sign of the charge, and $B_{0}$ refers to the total static field strength. From $\delta v_{i x}=\delta v_{e x}$ follows the constraint that

$\Omega_{i} \delta v_{i y}=\Omega_{e} \delta v_{e y}$.

The linearized projections of Ampère's law (4) are

$\kappa \delta B_{y}=\mu_{0} e n_{0}\left(\delta v_{i z}-\delta v_{e z}\right)$

$\kappa \delta B_{z}=\mu_{0} e n_{0}\left(\delta v_{e y}-\delta v_{i y}\right)$,

and, together with Eq. (A2), the first one yields

$\left[\kappa^{2} B_{0}-\mu_{0} e n_{0}\left(\Omega_{i}-\Omega_{e}\right)\right] \delta B_{y}=0$.

Hence either $\delta B_{y}=0$ or possible $\kappa$ values are restricted to

$\kappa_{c}=\frac{\sqrt{\omega_{p i}^{2}+\omega_{p e}^{2}}}{c}=\frac{\omega_{p}}{c}$,

where the species' plasma frequencies have been defined through $\omega_{p j}^{2}=n_{0} q_{j}^{2} / \varepsilon_{0} m_{j}$ and $\omega_{p}$ is the total plasma frequency given through $\omega_{p}^{2}=\omega_{p i}^{2}+\omega_{p e}^{2}$. In other words, there is no real range in $\kappa$ left, in which case there is no spectrum of evanescent modes. 
Let us first try to continue with $\delta B_{y} \neq 0$ but use $\kappa_{c}$. Then the combination of the $y$ component of Eq. (A1) with (A2) gives the relation

$\delta B_{y}=\frac{\omega_{p}^{3} V^{3}\left(\Omega_{i}+\Omega_{e}\right)}{c \cos \vartheta\left[c^{2} \Omega_{i}^{2} \Omega_{e}^{2}+\omega_{p}^{2} V^{2}\left(\Omega_{i}^{2}+\Omega_{i} \Omega_{e}+\Omega_{e}^{2}\right)\right]} \delta B_{z}$.

Using all the available information in the second Eq. (A3) yields

$$
\frac{c^{2} \Omega_{i}^{2} \Omega_{e}^{2}+\omega_{p}^{2} V^{2}\left(\Omega_{i}+\Omega_{e}\right)^{2}}{c^{2} \Omega_{i}^{2} \Omega_{e}^{2}+\omega_{p}^{2} V^{2}\left(\Omega_{i}^{2}+\Omega_{i} \Omega_{e}+\Omega_{e}^{2}\right)} \delta B_{z}=0 .
$$

Since the coefficient of $\delta B_{z}$ is strictly positive, we find that $\delta B_{z}=0$ and from Eq. (A6), also $\delta B_{y}=0$, i.e. no evanescent waves are possible.

Thus we backtrack to Eq. (A4), leave $\kappa$ as yet undetermined but proceed with $\delta B_{y}=0$. Now (A1) gives that

$\delta v_{j y}=-\frac{\kappa V^{2} \Omega_{j}}{B_{0}\left(\kappa^{2} V^{2}+\Omega_{j}^{2}\right)} \delta B_{z}$.

This has to obey Eq. (A2), which in turn gives

$\kappa V\left(\Omega_{i}^{2}-\Omega_{e}^{2}\right) \delta B_{z}=0$.

It is now clear that only the special case $\Omega_{i}^{2}=\Omega_{e}^{2}$, equivalent to $m_{i}=m_{e}$, will give a nonvanishing wave magnetic field, viz. in strict pair plasmas.

Discussing very briefly the pair plasma case, the remaining component of Eq. (A3) gives the required dispersion law for linear evanescent waves in pair plasmas,

$\kappa^{2}=\frac{\omega_{p}^{2}}{c^{2}}-\frac{\Omega^{2}}{V^{2}}$.

Here $\Omega=e B_{0} / m$ is the unique gyrofrequency in absolute value and $\omega_{p}$ is the total pair plasma frequency. It it seen that the modes in pair plasmas have wave magnetic fields that are linearly polarized along the z-axis, and, from the requirement that $\kappa^{2}>0$, are super-Alfvénic in the sense that $V^{2}>\Omega^{2} c^{2} / \omega_{p}^{2}=B_{0}^{2} / 2 \mu_{0} n_{0} m$.

\section{Appendix B}

\section{Elements of the pair plasmas treatment}

In pair plasmas $m_{i}=m_{e}=m$ and, as seen in the discussion following Eq. (13), $B_{y}=0$, so that Eqs. (9), (12) and (13) all give that $v_{i y}=-v_{e y}=v_{y}$. The subscript $i$ refers here to the positrons. What remains of the $z$ component of Ampère's law (4) indicates that $v_{i z}=v_{e z}=v_{z}$. Furthermore, Eq. (15) is now trivially obeyed and cannot be used to extract more conflicting information. The $y$ component of Ampère's law (4) reduces to

$\frac{d B_{z}}{d x}+2 \mu_{0} n e v_{y}=0$.

Substituting thus $v_{x}$ (and indirectly $n$ ) from Eq. (8), $v_{y}$ from Eq. (B1) and $v_{z}$ from the only remaining nonzero component of Eq. (9) into the energy integral Eq. (10) gives a single differential (not algebraic!) equation determining $B_{z}$. It is an energy-like integral for a particle with coordinate $B_{z}$ and unit mass (Verheest and Cattaert, 2005),

$\frac{1}{2}\left(\frac{d B_{z}}{d x}\right)^{2}+\psi\left(B_{z}\right)=0$,

moving in the pseudopotential

$$
\begin{aligned}
\psi\left(B_{z}\right)= & \frac{\mu_{0} n_{0} m \omega_{p}^{2} V^{2}\left(B_{z}-B_{z 0}\right)^{2}}{c^{2}\left(B_{z}^{2}-B_{z 0}^{2}-4 \mu_{0} n_{0} m V^{2}\right)^{2}} \times \\
& \times\left[\left(B_{z}+B_{z 0}\right)^{2}+4 B_{x 0}^{2}-8 \mu_{0} n_{0} m V^{2}\right] .
\end{aligned}
$$

The discussion is typical for the Sagdeev pseudopotential analysis (Verheest and Cattaert, 2005) and runs along lines found in many plasma wave treatments, as discussed specifically for pair plasmas by Decoster (1978).

Acknowledgements. Interesting discussions with T. Cattaert, M. A. Hellberg, I. Kourakis, G. S. Lakhina, S. Mahajan, T. Passot and Z. Yoshida are gratefully acknowledged. F. Verheest thanks the Fonds voor Wetenschappelijk Onderzoek (Vlaanderen) for a research grant.

Edited by: J. Büchner

Reviewed by: V. Berezhiani

\section{References}

Ablowitz, M. J. and Clarkson, P. A.: Solitons, Nonlinear Evolution Equations and Inverse Scattering, Cambridge University Press, Cambridge, UK, 1991.

Adlam, J. H. and Allen, J. E.: The structure of strong collision-free hydromagnetic waves, Philos. Mag., 3, 448-455, 1958.

Baumjohann, W., Treumann, R. A., Georgescu, E., Haerendel, G., Fornacon, K.-H., and Auster, U.: Wave form and packet structure of lion roars, Ann. Geophys., 17, 1528-1534, 1999, http://www.ann-geophys.net/17/1528/1999/.

Boehmer, H., Adams, M., and Rynn, N.: Positron trapping in a magnetic mirror configuration, Phys. Plasmas, 2, 4369-4371, 1995.

Cattaert, T. and Verheest, F.: Large amplitude parallel propagating electromagnetic oscillitons, Phys. Plasmas, 12, 012307, doi:10.1063/1.1824038, 2005.

Cattell, C. A., Dombeck, J., Wygant, J. R., Hudson, M. K., Mozer, F. S., Temerin, M. A., Peterson, W. K., Kletzing, C. A., Russell, C. T., and Pfaff, R. F.: Comparisons of Polar satellite observations of solitary wave velocities in the plasma sheet boundary and the high altitude cusp to those in the auroral zone, Geophys. Res. Lett., 26, 425-428, 1999. 
Cattell, C., Neiman, C., Dombeck, J., Crumley, J., Wygant, J., Kletzing, C. A., Peterson, W. K., Mozer, F. S., and André, M.: Large amplitude solitary waves in and near the Earth's magnetosphere, magnetopause and bow shock: Polar and Cluster observations, Nonlin. Processes Geophys., 10, 13-26, 2003,

http://www.nonlin-processes-geophys.net/10/13/2003/.

Chen, F. F.: Introduction to Plasma Physics, Plenum Press, New York, pp. 65-66, 1974.

Decoster, A.: Nonlinear travelling waves in a homogeneous cold collisionless plasma, Phys. Reports, 47, 285-422, 1978.

Dubinin, E., Sauer, K., and McKenzie, J. F.: Solitons and oscillitons in cold bi-ion plasmas: a parameter study, J. Plasma Phys., 68, 27-52, 2002.

Dubinin, E., Sauer, K., and McKenzie, J. F.: Nonlinear stationary whistler waves and whistler solitons (oscillitons). Exact solutions, J. Plasma Phys., 69, 305-330, 2003.

Ergun, R. E., Carlson, C. W., McFadden, J. P., Mozer, F. S., Delory, G. T., Peria, W., Chaston, C. C., Temerin, M., Roth, I., Muschietti, L., Elphic, R., Strangeway, R., Pfaff, R., Cattell, C. A., Klumpar, D., Shelley, E., Peterson, W., Möbius, E., and Kistler, L.: FAST satellite observations of large-amplitude solitary structures, Geophys. Res. Lett., 25, 2041-2044, 1998.

Franz, J. R., Kintner, P. M., and Pickett, J. S.: POLAR observations of coherent electric field structures, Geophys. Res. Lett., 25, 1277-1280, 1998.

Goldreich, P. and Julian, W. H.: Pulsar electrodynamics, Astrophys. J., 157, 869-880, 1969.

Hackenberg, P., Mann, G., and Marsch, E.: Solitons in multi-ion plasmas, J. Plasma Phys., 60, 845-859, 1998.

Hada, T., Kennel, C. F., and Buti, B.: Stationary nonlinear Alfén waves and solitons, J. Geophys. Res., 94, 65-77, 1989.

Hellberg, M. A., Baboolal, S., Mace, R. L., and Bharuthram, R.: The role of self-consistency in double-layer calculations, IEEE Trans. Plasma Sci., 20, 695-700, 1992.

Iwamoto, N.: Collective modes in nonrelativistic electron-positron plasmas, Phys. Rev. E, 47, 604-611, 1993.

Kivelson, M. G., Khurana, K. K., Walker, R. J., Warnecke, J., Russell, C. T., Linker, J. A., Southwood, D. J., and Polanskey, C.: Io's interaction with the Plasma Torus: Galileo magnetometer report, Science, 274, 396-398, 1996.

Lakhina, G. S. and Tsintsadze, N. L.: Large-amplitude lowfrequency electromagnetic waves in pulsar magnetospheres, Astrophys. Space Sci., 174, 143-150, 1990.

Mace, R. L., McKenzie, J. F., and Webb, G. M.: Conservation laws for steady flow and solitons in a multi-fluid plasma revisited, Phys. Plasmas, in press, 2007.

Matsumoto, H., Kojima, H., Miyatake, T., Omura, Y., Okada, M., Nagano, I., and Tsutsui M.: Electrostatic Solitary Waves (ESW) in the magnetotail: BEN wave forms observed by GEOTAIL, Geophys. Res. Lett., 21, 2915-2918, 1994.

McKenzie, J. F. and Doyle, T. B.: Oblique solitons in a cold magnetized plasma, Phys. Plasmas, 8, 4367-4374, 2001.

McKenzie, J. F., Dubinin, E., and Sauer, K.: Nonlinear waves propagating transverse to the magnetic field, J. Plasma Phys., 65, 213-233, 2001.

McKenzie, J. F., Dubinin, E., Sauer, K., and Doyle, T. B.: The application of the constants of the motion to nonlinear stationary waves in complex plasmas: a unified fluid dynamic point of view, J. Plasma Phys., 70, 431-462, 2004.
McKenzie, J. F., Dubinin, E. M., and Sauer, K.: Relativistic whistler oscillations - do they exist?, Nonlin. Processes Geophys., 12, 425-431, 2005,

http://www.nonlin-processes-geophys.net/12/425/2005/.

Oohara, W. and Hatakeyama, R.: Pair-ion plasma generation using fullerenes, Phys. Rev. Lett., 91, 205005, doi:10.1103/PhysRevLett.91.205005, 2003.

Ostriker, J. P. and Gunn, J. E.: On the nature of pulsars. I. Theory Astrophys. J., 157, 1395-1417, 1969.

Pacini, F.: Rotating neutron stars, pulsars and supernova remnants, Nature, 219, 145-146, 1968.

Paschmann, G., Haerendel, G., Sckopke, N., Möbius, E., Luhr, H., and Carlson, C. W.: Three-dimensional plasma structures with anomalous flow directions near the Earth's bow shock, J. Geophys. Res., 93, 11 279-11 294, 1988.

Petkaki, P. and Dougherty, M.: Waves close to the crossover frequency in the Jovian middle magnetosphere, Geophys. Res. Lett., 28, 211-214, 2001.

Pickett, J. S., Menietti, J. D., Gurnett, D. A., Tsurutani, B., Kintner, P. M., Klatt, E., and Balogh, A.: Solitary potential structures observed in the magnetosheath by the Cluster spacecraft, Nonlin. Processes Geophys., 10, 3-11, 2003, http://www.nonlin-processes-geophys.net/10/3/2003/.

Pickett, J. S., Kahler, S. W., Chen, L.-J., Huff, R. L., Santolik, O., Khotyaintsev, Y., Décréau, P. M. E., Winningham, D., Frahm, R., Goldstein, M. L., Lakhina, G. S., Tsurutani, B. T., Lavraud, B., Gurnett, D. A., André, M., Fazakerley, A., Balogh, A., and Rème, H.: Solitary waves observed in the auroral zone: the Cluster multi-spacecraft perspective, Nonlin. Processes Geophys., 11, 183-196, 2004,

http://www.nonlin-processes-geophys.net/11/183/2004/.

Pottelette, R., Ergun, R. E., Treumann, R. A., Berthomier, M., Carlson, C. W., McFadden, J. P., and Roth, I.: Modulated electronacoustic waves in auroral density cavities: FAST observations, Geophys. Res. Lett., 26, 2629-2632, 1999.

Rees, M. J.: New interpretation of extragalactic radio sources, Nature, 229, 312-317, 1971.

Rektorys, K. (Ed.): Survey of Applicable Mathematics, MIT Press, Cambridge, Mass., 1969.

Sagdeev, R.Z.: Cooperative phenomena and shock waves in collisionless plasmas, in: Reviews of Plasma Physics, vol. 4, edited by: Leontovich, M. A., Consultants Bureau, New York, pp. 23 91, 1966.

Sauer, K., Dubinin, E., and McKenzie, J. F.: New type of soliton in bi-ion plasmas and possible implications, Geophys. Res. Lett., 28, 3589-3592, 2001.

Sauer, K., Dubinin, E., and McKenzie, J. F.: Wave emission by whistler oscillitons: Application to "coherent lion roars", Geophys. Res. Lett., 29, 2226, doi:10.1029/2002GL015771, 2002.

Schwartz, S. J., Kessel, R. L., Brown, C. C., Woolliscroft, L. J. C., Dunlop, M. W., Farrugia, C. J., and Hall, D. S.: Active current sheets near the Earth's bow shock, J. Geophys. Res., 93, 11295 $11310,1988$.

Stasiewicz, K., Shukla, P. K., Gustafsson, G., Buchert, S., Lavraud, B., Thidé, B., and Klos, Z.: Slow magnetosonic solitons detected by the Cluster spacecraft, Phys. Rev. Lett., 90, 085002, doi:10.1103/PhysRevLett.90.085002, 2003a.

Stasiewicz, K., Longmore, M., Buchert, S., Shukla, P. K., Lavraud, B., and Pickett, J.: Properties of fast magnetosonic 
shocklets at the bow shock, Geophys. Res. Lett., 30, 2241, doi:10.1029/2003GL017971, 2003b.

Stenflo, L., Shukla, P. K., and Yu, M. Y.: Nonlinear propagation of electromagnetic waves in magnetized electron-positron plasmas, Astrophys. Space Sci., 117, 303-308, 1985.

Stix, T. H.: Waves in Plasmas, American Institute of Physics, New York, 1992.

Sturrock, P. A.: A model of pulsars, Astrophys. J., 164, 529-556, 1971.

Surko, C. M., Leventhal, M., and Passner, A.: Positron plasma in the laboratory, Phys. Rev. Lett., 62, 901-904, 1989.

Thomsen, M. F., Gosling, J. T., Bame, S. J., Quest, K. B., Russell, C. T., and Fuselier, S. A.: On the origin of hot diamagnetic cavities near the Earth's bow shock, J. Geophys. Res., 93, 11 311-11325, 1988.
Verheest, F., Cattaert, T., Dubinin, E., Sauer, K., and McKenzie, J. F.: Whistler oscillitons revisited: the role of charge neutrality?, Nonlin. Processes Geophys., 11, 447-452, 2004, http://www.nonlin-processes-geophys.net/11/447/2004/.

Verheest, F. and Cattaert, T.: Large amplitude solitary electromagnetic waves in electron-positron plasmas, Phys. Plasmas, 11, 3078-3082, 2004.

Verheest, F. and Cattaert, T.: Oblique propagation of large amplitude electromagnetic solitons in pair plasmas, Phys. Plasmas, 12, 032304, doi:10.1063/1.1853386, 2005.

Zank, G. P. and Greaves, R. G.: Linear and nonlinear modes in nonrelativistic electron-positron plasmas, Phys. Rev. E, 51, 60796090, 1995.

Zhang, Y., Matsumoto, H., and Kojima, H.: Lion roars in the magnetosheath: the Geotail observations, J. Geophys. Res., 103, 4615-4626, 1998. 